Research article

\title{
A checklist of succulent plants of Ahmedabad, Gujarat, India
}

\author{
Ruchi M. Patel ${ }^{1}$, Umerfaruq M. Qureshimatva ${ }^{2} *$, Rupesh R. Maurya ${ }^{2}$ \\ and Hitesh A. Solanki ${ }^{2}$ \\ ${ }^{1}$ Government Science College, Vankal, Mangrol Taliuka, Surat, Gujarat, India \\ ${ }^{2}$ Department of Botany, University School of Sciences, Gujarat University, Ahmedabad, Gujarat, India \\ *Corresponding Author: ufmqureshi@yahoo.in \\ [Accepted: 18 December 2016]

\begin{abstract}
The study deals with the diversity of succulent plants in Ahmedabad. Succulent plants are increasingly popular among plant collectors, home gardeners and professional landscapes for colorful leaves, sculptural shapes, simple care, etc. Succulents are widely used for the indoor gardening as well as outdoor gardening for their outstanding appearance. The study reported 73 species of succulent plants from Ahmedabad.
\end{abstract}

Keywords: Diversity - Succulent - Cactus - Ahmedabad.

[Cite as: Patel RM, Qureshimatva UM, Maurya RR \& Solanki HA (2016) A checklist of succulent plants of Ahmedabad, Gujarat, India. Tropical Plant Research 3(3): 686-693]

\section{INTRODUCTION}

Succulent plants have a global distribution and represented in nearly all habitat type. Over 30 botanical families have succulent plant species, ranging from tiny annuals plants to huge tree (IUCN 1997). Succulence is an adaptive response to drought, rapid drainage in rocky and sandy soil, high evaporation in windy, hot environments and in salty or alkaline habitats. There are probably more than five thousand species worldwide (Newton \& Chan 1998).

The "Succulent Karoo" of South Africa and Namibia boasts the richest succulent flora of Earth. Mexico is the country with the highest diversity of cactus in the American continent (Ortega \& Héctor 2006). More than 60 species are listed in the Red Data Book of the International Union for the Conservation of Nature (IUCN) (IUCN 2003). Many of these species has an outstanding biological, cultural, and economical importance. Several species of cactus are among the most dominant plants in different vegetation types, where they interact with a large variety of animal and plant species (Héctor et al. 2003).

All the succulents evolved from other related plants growing in a normal environment by adaptation to the changing climatic conditions of their habitat, especially the regularity and amount of rainfall. This process of adaptation varied in every family and doubtless many plants succumbed in the struggle for survival. Water is essential for the growth and life of all vegetation, including the succulents, which have mastered the art of economizing water (Rudolf 1980). In geological times, the earth's climate changed becoming drier as the mountains were pushed up to create rain shadows and deserts. Other plant families adapted similarly to these conditions and there are thousands of succulent species (Edwards \& Donoghue 2006).

Succulent plants are increasingly popular among plant collectors, home gardeners and professional landscapes for a number of reasons. With their colorful leaves, sculptural shapes and simple care, succulents are beautiful yet forgiving plant for pots (Debra 2010). Succuelents are highly diverse. The present study shows diversity in the succulents. They are highly ignored by taxonomist in gujarat just because many of them are ornamental. Now days succulents are used in outdoor and indoor gardening at various place like malls, industries, colleges, hospitals and gardens in the city of Ahmadabad. So there is urgent need to have documentation of such ornamental groups also.

\section{MATERIALS AND METHODS}

Study area

Ahmedabad is located $23.01^{\circ} \mathrm{N}$ Latitude and $72.61^{\circ}$ East Longitude covering $8,086.81 \mathrm{~km}^{2}$ area at an altitude of $55 \mathrm{~m}$ above the sea level (Qureshimatva et al. 2016). Ahmedabad has a hot semi-arid climate, with 
marginally less rain than required for a tropical savanna climate. There are three main seasons: summer, monsoon and winter. Aside from the monsoon season, the climate is extremely dry. The weather is hot through the months of March to June; the average summer maximum is $41^{\circ} \mathrm{C}\left(106^{\circ} \mathrm{F}\right)$, and the average minimum is $27^{\circ} \mathrm{C}$ $\left(81^{\circ} \mathrm{F}\right)$. From November to February, the average maximum temperature is $30^{\circ} \mathrm{C}\left(86^{\circ} \mathrm{F}\right)$, the average minimum is $15^{\circ} \mathrm{C}\left(59^{\circ} \mathrm{F}\right)$, and the climate is extremely dry. Cold northerly winds are responsible for a mild chill in January. The southwest monsoon brings a humid climate from mid-June to mid-September. The average annual rainfall is about 800 millimeters ( $31 \mathrm{in}$ ), but infrequent heavy torrential rains cause local rivers to flood and it is not uncommon for droughts to occur when the monsoon does not extend as far west as usual. The highest temperature recorded is $48.5^{\circ} \mathrm{C}\left(119.3^{\circ} \mathrm{F}\right)$ (Anonymous 2012).

Field work

Field wok was carried out during 2013 to 2014. The visits were conducted across various gardens; nursery besides theses also visited some people who has a personal collection of cactus and succulent. During field work the photos of each succulent were taken along with habit, stem, leaves and other floral parts. Field notes were taken to have information on habit, habitat and characteristics of succulents. All the specimens were identified with the help of available literature (Anderson 2012, Mary 2000, Qureshimatva 2016, Rudolf 1980, Scott 2012, Shah 1978).

\section{RESULTS AND DISCUSSION}

In the present study 45 genera and 73 species with 1 sub sp., 5 varieties and 2 cultivated varieties belonging to 15 families have been reported from the study area (Fig. 1; Table 1). In the present study 5 succulents were reported as indigenous and endemic to India. Other succulents were introduced from the Madagascar, Southern Africa, Brazil, Europe, Mexico, Tropical America, etc. (Fig. 2).

Table 1. List of succulent plants occurring in Ahmedabad.

\begin{tabular}{|c|c|c|c|c|c|c|}
\hline S.N. & Botanical Name & Family & $\begin{array}{l}\text { Common } \\
\text { Name }\end{array}$ & Native & $\begin{array}{l}\text { Propag } \\
\text { ation }\end{array}$ & Distribution \\
\hline 1 & Portulaca oleracea L. & Portulacaceae & $\begin{array}{l}\text { Purslane, } \\
\text { Lunia }\end{array}$ & Western Asia & $\begin{array}{l}\text { Stem } \\
\text { cutting }\end{array}$ & $\begin{array}{l}\text { Common in } \\
\text { Ahmedabad }\end{array}$ \\
\hline 2 & Portulaca pilosa $\mathrm{L}$. & Portulacaceae & Kiss me quick & $\begin{array}{l}\text { North } \\
\text { America }\end{array}$ & $\begin{array}{l}\text { stem } \\
\text { cutting }\end{array}$ & $\begin{array}{l}\text { Gurjar vaani, Gujarat } \\
\text { University Campus. }\end{array}$ \\
\hline 3 & $\begin{array}{l}\text { Commiphora wightii (Arn.) } \\
\text { Bhandari }\end{array}$ & Burseraceae & Gugal & Central Asia & $\begin{array}{l}\text { Seeds or } \\
\text { stem } \\
\text { cutting }\end{array}$ & Serenity \\
\hline 4 & Cissus quadrangularis L. & Vitaceae & $\begin{array}{l}\text { Veldt Grape, } \\
\text { Devil's } \\
\text { Backbone }\end{array}$ & $\begin{array}{l}\text { Southern } \\
\text { and eastern } \\
\text { Africa, } \\
\text { Arabia to } \\
\text { India }\end{array}$ & $\begin{array}{l}\text { Stem } \\
\text { cutting }\end{array}$ & $\begin{array}{l}\text { Gujarat University } \\
\text { Campus }\end{array}$ \\
\hline 5 & Cissus rotundifolia Vahl & Vitaceae & $\begin{array}{l}\text { Venezuelan } \\
\text { Treebine, } \\
\text { Arabian wax } \\
\text { cissus }\end{array}$ & Africa & $\begin{array}{l}\text { Stem } \\
\text { cutting }\end{array}$ & K. H. Bhatt's house. \\
\hline 6 & $\begin{array}{l}\text { Bryophyllum delagoense } \\
\text { (Eckl. \& Zeyh.) Druce }\end{array}$ & Crassulaceae & $\begin{array}{l}\text { Mother of } \\
\text { Millions }\end{array}$ & Madagascar & $\begin{array}{l}\text { Leaf } \\
\text { cutting }\end{array}$ & $\begin{array}{l}\text { Gujarat University } \\
\text { Campus }\end{array}$ \\
\hline 7 & $\begin{array}{l}\text { Bryophyllum fedtschenkoi } \\
\text { (Raym.-Hamet \& H.Perrier) } \\
\text { Lauz.-March. }\end{array}$ & Crassulaceae & $\begin{array}{l}\text { Air plant, } \\
\text { Cathedral } \\
\text { bells }\end{array}$ & $\begin{array}{l}\text { Madagascar } \\
\text { and Southern } \\
\text { Africa }\end{array}$ & $\begin{array}{l}\text { Leaf } \\
\text { cutting }\end{array}$ & $\begin{array}{l}\text { Common in } \\
\text { Ahmedabad }\end{array}$ \\
\hline 8 & $\begin{array}{l}\text { Kalanchoe blossfeldiana } \\
\text { Poelln }\end{array}$ & Crassulaceae & $\begin{array}{l}\text { Flaming Katy, } \\
\text { Christmas } \\
\text { Kalanchoe, } \\
\text { Florist } \\
\text { Kalanchoe }\end{array}$ & Madagascar & $\begin{array}{l}\text { Stem } \\
\text { cutting }\end{array}$ & $\begin{array}{l}\text { Common in } \\
\text { Ahmedabad }\end{array}$ \\
\hline
\end{tabular}


Patel et al. (2016) 3(3): 686-693

\begin{tabular}{|c|c|c|c|c|c|c|}
\hline 9 & $\begin{array}{l}\text { Kalanchoe tetraphylla } \mathrm{H} . \\
\text { Perrier }\end{array}$ & Crassulaceae & $\begin{array}{l}\text { Desert's } \\
\text { Cabbage } \\
\text { Paddle plant }\end{array}$ & South Africa & $\begin{array}{l}\text { Stem } \\
\text { cutting }\end{array}$ & $\begin{array}{l}\text { Gujarat University } \\
\text { Campus }\end{array}$ \\
\hline 10 & Kalanchoe tomentosa Baker & Crassulaceae & $\begin{array}{l}\text { Panda plant, } \\
\text { Plush plant, } \\
\text { Pussy ears. } \\
\text { chocolate } \\
\text { soldier }\end{array}$ & Madagascar & $\begin{array}{l}\text { Leaf } \\
\text { cutting }\end{array}$ & $\begin{array}{l}\text { Common in } \\
\text { Ahmedabad }\end{array}$ \\
\hline 11 & Sedum album $\mathrm{L}$. & Crassulaceae & $\begin{array}{l}\text { Coral Carpet, } \\
\text { White } \\
\text { stonecrop }\end{array}$ & Europe & $\begin{array}{l}\text { Stem } \\
\text { cutting }\end{array}$ & $\begin{array}{l}\text { Gujarat University } \\
\text { Campus }\end{array}$ \\
\hline 12 & $\begin{array}{l}\text { Sedum morganianum } \\
\text { E.Walther }\end{array}$ & Crassulaceae & $\begin{array}{l}\text { Burro's tail or } \\
\text { donkey tail }\end{array}$ & South Africa & $\begin{array}{l}\text { Stem } \\
\text { cutting }\end{array}$ & $\begin{array}{l}\text { Some private and } \\
\text { Home gardens }\end{array}$ \\
\hline 13 & $\begin{array}{l}\text { Acanthocereus tetragonus } \\
\text { (L.) Hummelinck }\end{array}$ & Cactaceae & $\begin{array}{l}\text { Triangle } \\
\text { cactus }\end{array}$ & $\begin{array}{l}\text { South Texas } \\
\text { to Venezuela, } \\
\text { on the } \\
\text { Atlantic } \\
\text { coast of } \\
\text { Central } \\
\text { America }\end{array}$ & $\begin{array}{l}\text { Seeds or } \\
\text { grafting }\end{array}$ & $\begin{array}{l}\text { Gujarat University } \\
\text { Campus }\end{array}$ \\
\hline 14 & $\begin{array}{l}\text { Astrophytum myriostigma } \\
\text { Lem }\end{array}$ & Cactaceae & $\begin{array}{l}\text { Bishop's Cap, } \\
\text { Bishop's Hat }\end{array}$ & $\begin{array}{l}\text { Highlands of } \\
\text { central and } \\
\text { northern } \\
\text { Mexico }\end{array}$ & $\begin{array}{l}\text { Seeds or } \\
\text { Grafting }\end{array}$ & K. H. Bhatt's house. \\
\hline 15 & $\begin{array}{l}\text { Brasiliopuntia brasiliensis } \\
\text { (Willd.) A.Berger }\end{array}$ & Cactaceae & $\begin{array}{l}\text { Brazilian } \\
\text { Prickly Pear }\end{array}$ & Brazil & $\begin{array}{l}\text { Seeds and } \\
\text { stem } \\
\text { cuttings }\end{array}$ & $\begin{array}{l}\text { Gujarat University } \\
\text { Campus }\end{array}$ \\
\hline 16 & Cereus hexagonus (L.) Mill & Cactaceae & $\begin{array}{l}\text { Hedge cactus, } \\
\text { spiny tree } \\
\text { cactus }\end{array}$ & $\begin{array}{l}\text { South } \\
\text { America }\end{array}$ & $\begin{array}{l}\text { Stem } \\
\text { cutting }\end{array}$ & K. H. Bhatt's house. \\
\hline 17 & Echinocactus grusonii Hildm. & Cactaceae & $\begin{array}{l}\text { Golden Barrel } \\
\text { Cactus }\end{array}$ & Mexico & Seeds & K. H. Bhatt's house. \\
\hline 18 & Echinocactus texensis Hopffe & Cactaceae & $\begin{array}{l}\text { Horse } \\
\text { Crippler, } \\
\text { Devil's head }\end{array}$ & Mexico & Seeds & $\begin{array}{l}\text { Gujarat University } \\
\text { Campus }\end{array}$ \\
\hline 19 & $\begin{array}{l}\text { Epiphyllum anguliger (Lem.) } \\
\text { G.Don }\end{array}$ & Cactaceae & $\begin{array}{l}\text { Rickrack } \\
\text { Cactus }\end{array}$ & Mexico & $\begin{array}{l}\text { Leaf } \\
\text { cutting }\end{array}$ & $\begin{array}{l}\text { The Sarabhai } \\
\text { Foundation Botanical } \\
\text { Garden }\end{array}$ \\
\hline 20 & $\begin{array}{l}\text { Epiphyllum oxypetalum (DC.) } \\
\text { Haw. }\end{array}$ & Cactaceae & Brahmakamal & Mexico & $\begin{array}{l}\text { Rhizome, } \\
\text { herbaceous } \\
\text { stem and } \\
\text { leaf cutting } \\
\text { or layering }\end{array}$ & Private gardens \\
\hline 21 & $\begin{array}{l}\text { Ferocactus peninsulae } \\
\text { (A.A.Weber) Britton \& Rose }\end{array}$ & Cactaceae & Barral cactus & Mexico & Seeds & Gurjar vaani \\
\hline 22 & Hamatocactus sp. & Cactaceae & Turk's head & $\begin{array}{l}\text { Native of } \\
\text { Argentina } \\
\text { and Paraguay }\end{array}$ & $\begin{array}{l}\text { Seed or } \\
\text { cutting }\end{array}$ & $\begin{array}{l}\text { Dept. of Botany } \\
\text { Gujarat University }\end{array}$ \\
\hline 23 & $\begin{array}{l}\text { Harrisia martinii (Labour.) } \\
\text { Britton }\end{array}$ & Cactaceae & $\begin{array}{l}\text { Harrisia } \\
\text { cactus, } \\
\text { Moonlight } \\
\text { cactus }\end{array}$ & $\begin{array}{l}\text { Argentina } \\
\text { and Paraguay }\end{array}$ & $\begin{array}{l}\text { Stem } \\
\text { cutting }\end{array}$ & $\begin{array}{l}\text { Gujarat University } \\
\text { Campus }\end{array}$ \\
\hline
\end{tabular}


Patel et al. (2016) 3(3): 686-693

\begin{tabular}{|c|c|c|c|c|c|c|}
\hline 24 & $\begin{array}{l}\text { Mammillaria beneckei } \\
\text { Ehrenb }\end{array}$ & Cactaceae & Mammillaria & Mexico & Seeds & $\begin{array}{l}\text { Gujarat University } \\
\text { Campus }\end{array}$ \\
\hline 25 & $\begin{array}{l}\text { Myrtillocactus geometrizans } \\
\text { (Mart. ex Pfeiff.) Console }\end{array}$ & Cactaceae & Blue Candle & $\begin{array}{l}\text { Northern } \\
\text { central } \\
\text { Mexico } \\
\text { down to } \\
\text { Oaxaca }\end{array}$ & $\begin{array}{l}\text { Seeds, } \\
\text { cuttings in } \\
\text { summer }\end{array}$ & $\begin{array}{l}\text { Gujarat University } \\
\text { Campus }\end{array}$ \\
\hline 26 & $\begin{array}{l}\text { Opuntia cochenillifera }(\mathrm{L} .) \\
\text { Miller }\end{array}$ & Cactaceae & $\begin{array}{l}\text { Cochineal } \\
\text { nopal cactus, } \\
\text { warm hand }\end{array}$ & Mexico & $\begin{array}{l}\text { Seeds or } \\
\text { stem } \\
\text { cutting }\end{array}$ & Serenity Library, Bhat \\
\hline 27 & $\begin{array}{l}\text { Opuntia cylindrica (Lam.) } \\
\text { DC }\end{array}$ & Cactaceae & Cane cactus & $\begin{array}{l}\text { Ecuador and } \\
\text { Per }\end{array}$ & $\begin{array}{l}\text { Stem } \\
\text { cutting }\end{array}$ & $\begin{array}{l}\text { Private garden near } \\
\text { Sardar Stadium, Gujarat } \\
\text { University Campus }\end{array}$ \\
\hline 28 & Opuntia elatior Mill & Cactaceae & $\begin{array}{l}\text { Prickly Pear, } \\
\text { Slipper Thorn, } \\
\text { Phafdo Thor }\end{array}$ & $\begin{array}{l}\text { Central } \\
\text { America }\end{array}$ & $\begin{array}{l}\text { Seeds or } \\
\text { stem } \\
\text { cutting }\end{array}$ & $\begin{array}{l}\text { Gujarat University } \\
\text { Campus }\end{array}$ \\
\hline 29 & $\begin{array}{l}\text { Opuntia microdasys (Lehm.) } \\
\text { Pfeiff }\end{array}$ & Cactaceae & $\begin{array}{l}\text { Branching } \\
\text { Beavertail }\end{array}$ & Mexico & $\begin{array}{l}\text { Stem } \\
\text { cutting or } \\
\text { Seeds }\end{array}$ & $\begin{array}{l}\text { Serenity, Gujarat } \\
\text { University Campus }\end{array}$ \\
\hline 30 & Pereskia grandifolia Haw & Cactaceae & Rose cactus & $\begin{array}{l}\text { Brazil } \\
\text { (Uncertain) }\end{array}$ & $\begin{array}{l}\text { Cutting or } \\
\text { seed }\end{array}$ & Serenity \\
\hline 31 & $\begin{array}{l}\text { Schlumbergera kautskyi } \\
\text { (Horobin \& McMillan) } \\
\text { N.P.Taylor }\end{array}$ & Cactaceae & $\begin{array}{l}\text { Christmas } \\
\text { Cactus }\end{array}$ & $\begin{array}{l}\text { Brazil } \\
\text { (Espírito } \\
\text { Santo, Minas } \\
\text { Gerais) }\end{array}$ & $\begin{array}{l}\text { Stem } \\
\text { cutting }\end{array}$ & Private gardens \\
\hline 32 & $\begin{array}{l}\text { Aptenia cordifolia (L.f. ) } \\
\text { Schwantes }\end{array}$ & Cactaceae & $\begin{array}{l}\text { Baby Sun } \\
\text { Rose }\end{array}$ & $\begin{array}{l}\text { North } \\
\text { America }\end{array}$ & $\begin{array}{l}\text { Stem } \\
\text { cutting }\end{array}$ & Private gardens \\
\hline 33 & $\begin{array}{l}\text { Adenium obesum Roem. \& } \\
\text { Schult }\end{array}$ & Apocynaceae & Desert rose & Africa & $\begin{array}{l}\text { Seeds, } \\
\text { grafting } \\
\text { and cutting }\end{array}$ & K. H. Bhatt's house. \\
\hline 34 & Pachypodium lamerei Drake & Apocynaceae & $\begin{array}{l}\text { Madagascar } \\
\text { Palm, Club } \\
\text { Foot }\end{array}$ & Madagascar & Seeds & $\begin{array}{l}\text { Science city garden } \\
\text { and K. H. Bhatt's } \\
\text { house. }\end{array}$ \\
\hline 35 & Plumeria alba $\mathrm{L}$. & Apocynaceae & $\begin{array}{l}\text { White } \\
\text { champa }\end{array}$ & $\begin{array}{l}\text { South } \\
\text { America }\end{array}$ & Seed & $\begin{array}{l}\text { Gujarat University } \\
\text { Campus }\end{array}$ \\
\hline 36 & Plumeria rubra $\mathrm{L}$. & Apocynaceae & Champa & America & Seeds & $\begin{array}{l}\text { Gujarat University } \\
\text { Campus }\end{array}$ \\
\hline 37 & $\begin{array}{l}\text { Ceropegia bulbosa Roxb. } \\
\text { var. lushii (Grah.) Hook.f. }\end{array}$ & Asclepiadaceae & $\begin{array}{l}\text { Bulbous } \\
\text { Ceropegia }\end{array}$ & Africa & $\begin{array}{l}\text { Nodal } \\
\text { segments }\end{array}$ & $\begin{array}{l}\text { Gujarat University } \\
\text { Campus }\end{array}$ \\
\hline 38 & Sarcostemma acidum Voigt & Asclepiadaceae & Soma & $\begin{array}{l}\text { India and } \\
\text { Africa }\end{array}$ & Seed & $\begin{array}{l}\text { Serenity Library, } \\
\text { Bhat. }\end{array}$ \\
\hline 39 & Bacopa monnieri (L.) Wettst & Scrophulariaceae & $\begin{array}{l}\text { Brahmi, } \\
\text { Baam }\end{array}$ & India & $\begin{array}{l}\text { Stem } \\
\text { cutting }\end{array}$ & $\begin{array}{l}\text { Gujarat University } \\
\text { Campus }\end{array}$ \\
\hline 40 & Basella alba L. & Basellaceae & $\begin{array}{l}\text { Red vine } \\
\text { spinach }\end{array}$ & Asia & Seed & $\begin{array}{l}\text { Gujarat University } \\
\text { Campus }\end{array}$ \\
\hline 41 & $\begin{array}{l}\text { Alluaudia procera (Drake) } \\
\text { Drake }\end{array}$ & Didiereaceae & $\begin{array}{l}\text { Madagascan } \\
\text { Ocotillo }\end{array}$ & $\begin{array}{l}\text { Southern \& } \\
\text { south-western } \\
\text { Madagascar } \\
\text { (Toliara) }\end{array}$ & $\begin{array}{l}\text { Stem } \\
\text { cutting }\end{array}$ & $\begin{array}{l}\text { Gujarat University } \\
\text { Campus }\end{array}$ \\
\hline
\end{tabular}




\begin{tabular}{|c|c|c|c|c|c|c|}
\hline 42 & $\begin{array}{l}\text { Aemaralmeidia fusiformis } \\
\text { (Buch.-Ham) S.M. Almeida } \\
\text { \& S. Dutta ex Santosh Yadav } \\
\text { \& Rashmi Sharma }\end{array}$ & Euphorbiaceae & Thor & - & $\begin{array}{l}\text { Stem } \\
\text { cutting }\end{array}$ & $\begin{array}{l}\text { Gujarat University } \\
\text { Campus }\end{array}$ \\
\hline 43 & Euphorbia antiquorum L. & Euphorbiaceae & Thor & $\begin{array}{l}\text { Peninsular } \\
\text { India }\end{array}$ & Seed & $\begin{array}{l}\text { Common in } \\
\text { Ahmedabad }\end{array}$ \\
\hline 44 & $\begin{array}{l}\text { Euphorbia cristata B.Heyne } \\
\text { ex Roth }\end{array}$ & Euphorbiaceae & $\begin{array}{l}\text { Thor, } \\
\text { Neurang }\end{array}$ & - & $\begin{array}{l}\text { Stem } \\
\text { cutting }\end{array}$ & Science city \\
\hline 45 & Euphorbia milii Des Moul. & Euphorbiaceae & Thor & - & $\begin{array}{l}\text { Stem } \\
\text { cutting }\end{array}$ & $\begin{array}{l}\text { Gujarat University } \\
\text { Campus, St. Xavier } \\
\text { College Campus }\end{array}$ \\
\hline 46 & $\begin{array}{l}\text { Euphorbia milii var. } \\
\text { splendens (Bojer ex Hook.) } \\
\text { Ursch \& Leandri }\end{array}$ & Euphorbiaceae & $\begin{array}{l}\text { Crown of } \\
\text { thorns }\end{array}$ & & $\begin{array}{l}\text { Stem } \\
\text { cutting }\end{array}$ & $\begin{array}{l}\text { Gujarat University } \\
\text { Campus, St. Xavier } \\
\text { College Campus. }\end{array}$ \\
\hline 47 & Euphorbia neriifolia L. & Euphorbiaceae & $\begin{array}{l}\text { Thor, } \\
\text { Neurang }\end{array}$ & India & $\begin{array}{l}\text { Stem } \\
\text { cutting }\end{array}$ & $\begin{array}{l}\text { Common in } \\
\text { Ahmedabad }\end{array}$ \\
\hline 48 & Jatropha podagrica Hooker & Euphorbiaceae & $\begin{array}{l}\text { Buddha belly } \\
\text { plant, Bottle } \\
\text { plant shrub }\end{array}$ & $\begin{array}{l}\text { Tropical } \\
\text { America }\end{array}$ & Seeds & $\begin{array}{l}\text { Common in } \\
\text { Ahmedabad }\end{array}$ \\
\hline 49 & $\begin{array}{l}\text { Pedilanthus tithymaloides } \\
\text { (Linn.) Poit }\end{array}$ & Euphorbiaceae & $\begin{array}{l}\text { Nivali, } \\
\text { Vilayati-sher }\end{array}$ & $\begin{array}{l}\text { Tropical } \\
\text { America }\end{array}$ & $\begin{array}{l}\text { Stem } \\
\text { cutting }\end{array}$ & $\begin{array}{l}\text { Common in } \\
\text { Ahmedabad }\end{array}$ \\
\hline 50 & $\begin{array}{l}\text { Pedilanthus tithymaloides } \\
\text { subsp. smallii (Millsp.) } \\
\text { Dressler }\end{array}$ & Euphorbiaceae & $\begin{array}{l}\text { Nivali, } \\
\text { Vilayati-she }\end{array}$ & - & $\begin{array}{l}\text { Stem } \\
\text { cutting }\end{array}$ & $\begin{array}{l}\text { Common in } \\
\text { Ahmedabad }\end{array}$ \\
\hline 51 & $\begin{array}{l}\text { Pedilanthus tithymaloides } \\
\text { var. varigatus (L.) Poit }\end{array}$ & Euphorbiaceae & $\begin{array}{l}\text { Nivali, } \\
\text { Vilayati-sher }\end{array}$ & - & $\begin{array}{l}\text { Stem } \\
\text { cutting }\end{array}$ & Law garden \\
\hline 52 & Synadenium grantii Hook.f. & Euphorbiaceae & $\begin{array}{l}\text { African Milk } \\
\text { Bush }\end{array}$ & $\begin{array}{l}\text { East Central } \\
\text { Africa. }\end{array}$ & $\begin{array}{l}\text { Stem } \\
\text { cutting }\end{array}$ & Private garden \\
\hline 53 & Tirucalia indica Raf. & Euphorbiaceae & $\begin{array}{l}\text { Sher, Indian } \\
\text { Tree Spurge, } \\
\text { saptala (in } \\
\text { Sanskrit) }\end{array}$ & South Africa & $\begin{array}{l}\text { Stem } \\
\text { cutting }\end{array}$ & $\begin{array}{l}\text { Private garden near } \\
\text { SG highway }\end{array}$ \\
\hline 54 & Aechmea distichantha Lem & Bromeliaceae & $\begin{array}{l}\text { Brazilian } \\
\text { vaseplant }\end{array}$ & Brazil & Offsets & Private garden \\
\hline 55 & $\begin{array}{l}\text { Aechmea fasciata (Lindl.) } \\
\text { Baker }\end{array}$ & Bromeliaceae & $\begin{array}{l}\text { Silver vase } \\
\text { plant }\end{array}$ & Brazil & Offsets & $\begin{array}{l}\text { Gujarat University } \\
\text { Campus }\end{array}$ \\
\hline 56 & $\begin{array}{l}\text { Deuterocohnia scapigera } \\
\text { (Rauh \& L.Hrom.) } \\
\text { M.A.Spencer \& L.B.Sm. }\end{array}$ & Bromeliaceae & - & - & Seed & Private garden \\
\hline 57 & Agave americana L. & Agavaceae & $\begin{array}{l}\text { Century plant, } \\
\text { American aloe }\end{array}$ & Mexico & Suckers & $\begin{array}{l}\text { Common in } \\
\text { Ahmedabad }\end{array}$ \\
\hline 58 & $\begin{array}{l}\text { Agave sisalana Perrine ex } \\
\text { Engelm }\end{array}$ & Agavaceae & $\begin{array}{l}\text { Baby Sun } \\
\text { Rose, }\end{array}$ & Mexico & Suckers & $\begin{array}{l}\text { Common in } \\
\text { Ahmedabad }\end{array}$ \\
\hline 59 & $\begin{array}{l}\text { Agave victoriae-reginae } \\
\text { T.Moore }\end{array}$ & Agavaceae & $\begin{array}{l}\text { Baby Sun } \\
\text { Rose }\end{array}$ & Mexico & $\begin{array}{l}\text { Seeds or } \\
\text { Suckers }\end{array}$ & $\begin{array}{l}\text { Common in } \\
\text { Ahmedabad }\end{array}$ \\
\hline 60 & Agave vivipara $\mathrm{L}$. & Agavaceae & $\begin{array}{l}\text { Caribbean } \\
\text { Century Plant }\end{array}$ & - & Suckers & Serenity \\
\hline
\end{tabular}


Patel et al. (2016) 3(3): 686-693

\begin{tabular}{|c|c|c|c|c|c|c|}
\hline 61 & $\begin{array}{l}\text { Nolina recurvata (Lem.) } \\
\text { Hemsl }\end{array}$ & Agavaceae & $\begin{array}{l}\text { Pony Tail } \\
\text { Palm }\end{array}$ & $\begin{array}{l}\text { Tamaulipas } \\
\text { (Mexico) }\end{array}$ & Seeds & K. H. Bhatt's house. \\
\hline 62 & Yuccaa loifolia $\mathrm{L}$. & Agavaceae & $\begin{array}{l}\text { Spanish } \\
\text { bayonet }\end{array}$ & - & Seeds & $\begin{array}{l}\text { Gujarat University } \\
\text { Campus }\end{array}$ \\
\hline 63 & $\begin{array}{l}\text { Sansevieria cylindrica Bojer } \\
\text { ex Hook }\end{array}$ & Sansevieriaceae & Sasevieria & $\begin{array}{l}\text { Native to } \\
\text { Tropical Asia } \\
\text { and natal }\end{array}$ & $\begin{array}{l}\text { Leaf } \\
\text { cutting }\end{array}$ & $\begin{array}{l}\text { Gujarat University } \\
\text { Campus }\end{array}$ \\
\hline 64 & $\begin{array}{l}\text { Sansevieria ehrenbergii } \\
\text { Schweinf. ex Baker }\end{array}$ & Sansevieriaceae & $\begin{array}{l}\text { Blue } \\
\text { sansevieria, } \\
\text { sword } \\
\text { sansevieria }\end{array}$ & Africa & $\begin{array}{l}\text { Leaf } \\
\text { cutting }\end{array}$ & Serenity \\
\hline 65 & $\begin{array}{l}\text { Sanseneria hyacinthoides (L.) } \\
\text { Hort ex Staud }\end{array}$ & Sansevieriaceae & Piles root & Africa & $\begin{array}{l}\text { Leaf } \\
\text { cutting }\end{array}$ & $\begin{array}{l}\text { Gujarat University } \\
\text { Campus }\end{array}$ \\
\hline 66 & Sansevieria kirkii Baker & Sansevieriaceae & Snake plant & $\begin{array}{l}\text { Kenya } \\
\text { (Africa) }\end{array}$ & $\begin{array}{l}\text { Leaf } \\
\text { cutting }\end{array}$ & Serenity \\
\hline 67 & Sansevieria trifasciata Prain & Sansevieriaceae & $\begin{array}{l}\text { Mother-in- } \\
\text { law's tongue }\end{array}$ & Africa & $\begin{array}{l}\text { Leaf } \\
\text { cutting }\end{array}$ & $\begin{array}{l}\text { Gujarat University } \\
\text { Campus }\end{array}$ \\
\hline 68 & $\begin{array}{l}\text { Sansevieria trifasciata cv } \\
\text { golden hahnii }\end{array}$ & Sansevieriaceae & $\begin{array}{l}\text { Mother-in- } \\
\text { law's tongue }\end{array}$ & Africa & $\begin{array}{l}\text { Leaf } \\
\text { cutting }\end{array}$ & Private gardens \\
\hline 69 & $\begin{array}{l}\text { Sansevieria trifasciata cv } \\
\text { hahnii Graff }\end{array}$ & Sansevieriaceae & $\begin{array}{l}\text { Mother-in- } \\
\text { law's tongue }\end{array}$ & Africa & $\begin{array}{l}\text { Leaf } \\
\text { cutting }\end{array}$ & Private gardens \\
\hline 70 & $\begin{array}{l}\text { Sansevieria trifasciata var. } \\
\text { laurentii (De Wild.) N.E.Br }\end{array}$ & Sansevieriaceae & $\begin{array}{l}\text { Mother-in- } \\
\text { law's tongue }\end{array}$ & $\begin{array}{l}\text { Belgian } \\
\text { Congo }\end{array}$ & $\begin{array}{l}\text { Leaf } \\
\text { cutting }\end{array}$ & $\begin{array}{l}\text { Gujarat University } \\
\text { Campus }\end{array}$ \\
\hline 71 & Aloe deltoideodonta Baker & Aloeaceae & $\begin{array}{l}\text { Baby Sun } \\
\text { Rose }\end{array}$ & Madagascar & Offshoots & Private gardens \\
\hline 72 & Aloe maculata All & Aloeaceae & $\begin{array}{l}\text { Baby Sun } \\
\text { Rose }\end{array}$ & South Africa & $\begin{array}{l}\text { Rhizome, } \\
\text { tubers, } \\
\text { corns or } \\
\text { bulbs }\end{array}$ & Private gardens \\
\hline 73 & Aloe vera (L.) Burm. f. & Aloeaceae & $\begin{array}{l}\text { Baby Sun } \\
\text { Rose }\end{array}$ & South Africa & Offshoots & $\begin{array}{l}\text { Common in } \\
\text { Ahmedabad }\end{array}$ \\
\hline 74 & $\begin{array}{l}\text { Gasteria batesiana } \\
\text { G.D.Rowley }\end{array}$ & Aloeaceae & - & South Africa & Offshoots & Serenity \\
\hline 75 & $\begin{array}{l}\text { Gasteria obliqua (Aiton) } \\
\text { Duval. }\end{array}$ & Aloeaceae & - & $\begin{array}{l}\text { South Africa, } \\
\text { Lesotho and } \\
\text { Swaziland. }\end{array}$ & Offshoots & Serenity \\
\hline 76 & $\begin{array}{l}\text { Haworthia coarctata var. } \\
\text { adelaidensis (Poelln.) } \\
\text { M.B.Bayer }\end{array}$ & Aloeaceae & - & South Africa & Offshoots & K. H. Bhatt's house. \\
\hline 77 & $\begin{array}{l}\text { Haworthia fasciata (Willd.) } \\
\text { Haw }\end{array}$ & Aloeaceae & $\begin{array}{l}\text { Variegated } \\
\text { Zebra plant }\end{array}$ & South Africa & $\begin{array}{l}\text { Offsets, } \\
\text { leaves }\end{array}$ & K. H. Bhatt's house. \\
\hline 78 & Haworthia glauca Baker & Aloeaceae & - & $\begin{array}{l}\text { Eastern Cape } \\
\text { of South } \\
\text { Africa }\end{array}$ & $\begin{array}{l}\text { Seeds and } \\
\text { offsets }\end{array}$ & Private gardens \\
\hline 79 & Haworthia limifolia Marloth & Aloeaceae & $\begin{array}{l}\text { File Leafed } \\
\text { Haworthia }\end{array}$ & South Africa & $\begin{array}{l}\text { Seeds, } \\
\text { Offsets or } \\
\text { leaf } \\
\text { cutting }\end{array}$ & $\begin{array}{l}\text { Shraddha house and } \\
\text { Serenity }\end{array}$ \\
\hline
\end{tabular}



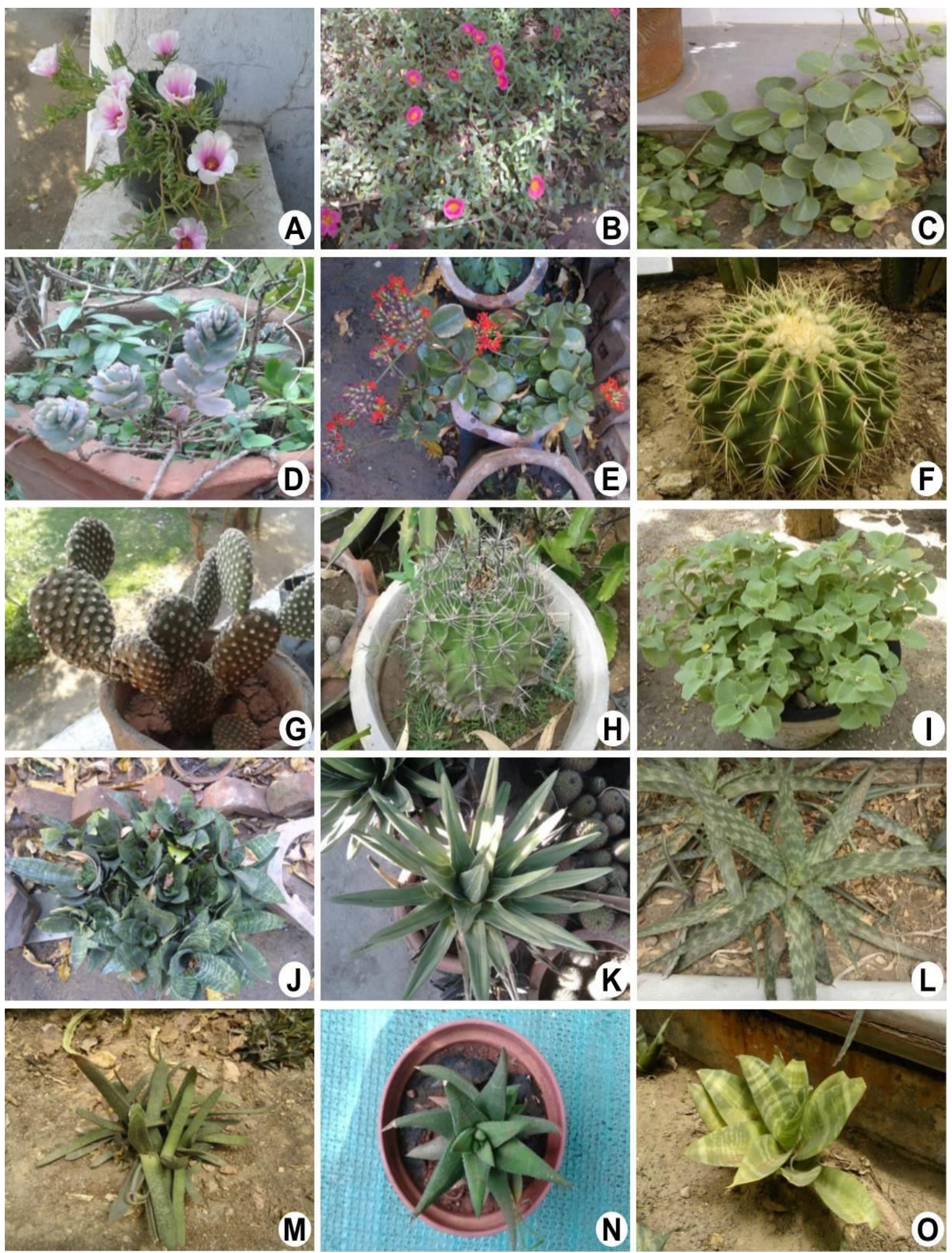

Figure 1. Photographs of some succulents: A, Portulaca pilosa Linn. Spp. Grandiflora(Hook.) Gesink; B, Portulaca pilosa L.; C, Cissus rotundifolia Vahl; D, Bryophyllum fedtschenkoi (Raym.-Hamet \& H.Perrier) Lauz.-March; E, Kalanchoe blossfeldiana Poelln; F, Echinocactus grusonii Hildm; G, Opuntia microdasys (Lehm.) Pfeiff.; H, Ferocactus peninsulae (A.A.Weber) Britton \& Rose; I, Plectranthus amboinicus (Lour.) Spreng; J, Aechmea fasciata (Lindl.) Baker.; K, Agave victoriae-reginae T.Moore Gard.; L, Aloe maculata All.

\section{ACKNOWLEDGEMENTS}

Authors are thankful to Head of the Department of Botany, Dr. A. U. Mankad and Dr. Santosh L. Yadav, Chief Botanist, The Serenity Library for their constant help and support. 


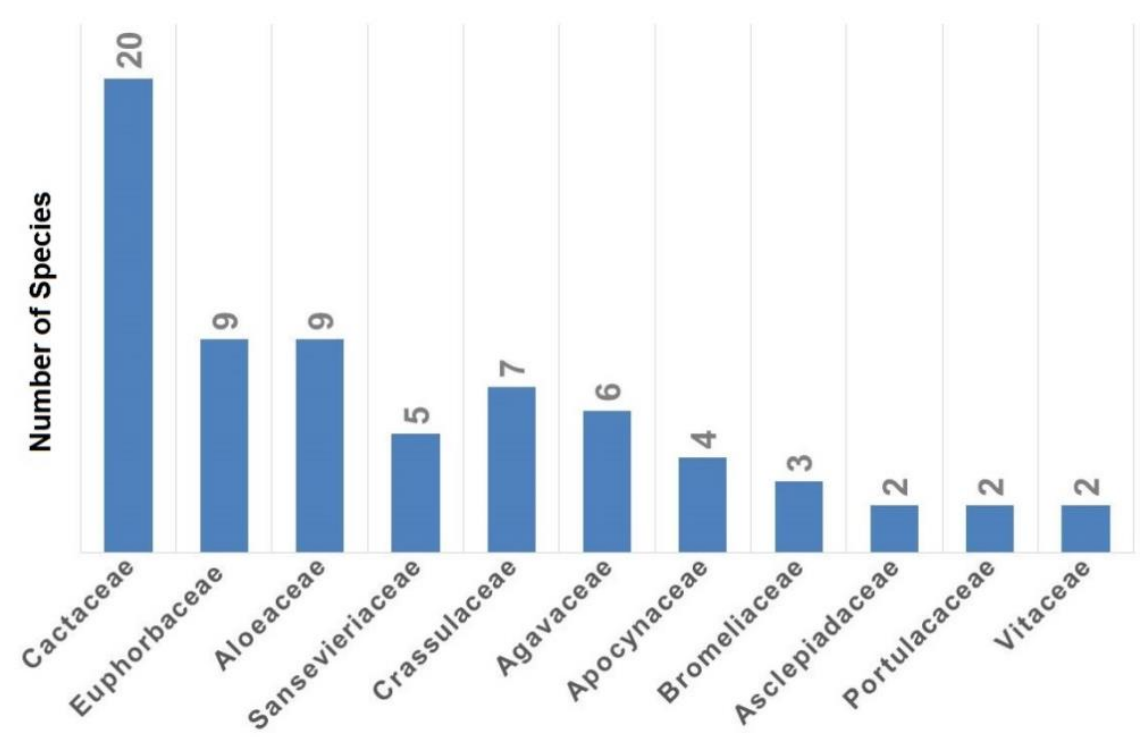

Figure 2. Number of succulent species in dominant families.

\section{REFERENCES}

Anderson M (2012) The Complete Illustrated Guide to Growing Cacti \& Succulents: the Definitive Practical Reference on identification, Care and Cultivation, with a Directory of 400 Varieties and 700 Photographs. Anness Publishing.

Anonymous (2012) Hong Kong Observatory.

Debra LB (2010) Succulent Container Gardens: Design Eye-Catching Displays with 350 Easy-care plants. Timber Press, Portland.

Edward FA (2001) The Cactus Family. Portland, Ore. Timber Press, United States of America.

Edwards EJ \& Donoghue MJ (2006) Pereskia and the Origin of the Cactus Life- Form. The American Naturalist 167(6): 777-793.

Héctor G-Á, Teresa V \& Pablo O-B (2003) Demographic trends in the Cactaceae. The Botanical Review 69: 173-203.

IUCN (1997) Cactus and Succulent Plants. International Union for the Conservation of Nature and Natural Resources. Available from: http://www.iucnredlist.org/ (accessed: 16 Mar. 2014)

IUCN (2003) Red List of Threatened Species. International Union for the Conservation of Nature and Natural Resources. Available from: http://www.iucnredlist.org/ (accessed: 16 Mar. 2014)

Mary I (2000) Agave, Yuccas and Related Plants: A Gardener "s Guide. Timber Press, United States of America.

Newton DJ \& Chan J (1998) South Africa's Trade in Southern African Succulent Plants. TRAFFIC East/Southern Africa, Johannesburg, Republic of South Africa.

Ortega BP \& Héctor G-Á (2006) Global diversity and conservation priorities in the Cactaceae. Biodiversity and Conservation 15: 817-827.

Qureshimatva UM, Gamit SB, Maurya RR, Solanki HA \& Yadav SL (2016) Cheklist of palms in Ahmedabad. Gujarat, India. International Journal of Recent Scientific Research 7(3): 9413-9417.

Rudolf S (1980) London Cacti and Succulents. The Hamlyn Publishing Group Ltd., U.K.

Scott DC (2012) The Gardener's Guide to Cactus: The 100 Best Paddles, Barrels, Columns, and Globes. Timber Press, United States of America.

Shah GL (1978) Flora of Gujarat State. Sardar Patel University, Vallabh, Vidyanagar, Anand, Gujarat. 